\title{
Genome-wide association studies of fertility and calving traits in Brown Swiss cattle using imputed whole-genome sequences
}

Mirjam Frischknecht ${ }^{1,2^{*}}$ (D), Beat Bapst ${ }^{1}$, Franz R. Seefried ${ }^{1}$, Heidi Signer-Hasler ${ }^{2}$, Dorian Garrick ${ }^{3}$, Christian Stricker ${ }^{4}$, Intergenomics Consortium ${ }^{5}$, Ruedi Fries ${ }^{6}$, Ingolf Russ ${ }^{7}$, Johann Sölkner ${ }^{8}$, Anna Bieber $^{9}$, Maria G. Strillacci ${ }^{10}$, Birgit Gredler-Grandl ${ }^{1}$ and Christine Flury ${ }^{2}$

\begin{abstract}
Background: The detection of quantitative trait loci has accelerated with recent developments in genomics. The introduction of genomic selection in combination with sequencing efforts has made a large amount of genotypic data available. Functional traits such as fertility and calving traits have been included in routine genomic estimation of breeding values making large quantities of phenotypic data available for these traits. This data was used to investigate the genetics underlying fertility and calving traits and to identify potentially causative genomic regions and variants.

We performed genome-wide association studies for 13 functional traits related to female fertility as well as for direct and maternal calving ease based on imputed whole-genome sequences. Deregressed breeding values from 1000-5000 bulls per trait were used to test for associations with approximately 10 million imputed sequence SNPs.

Results: We identified a QTL on BTA17 associated with non-return rate at 56 days and with interval from first to last insemination. We found two significantly associated non-synonymous SNPs within this QTL region. Two more QTL for fertility traits were identified on BTA25 and 29. A single QTL was identified for maternal calving traits on BTA13 whereas three QTL on BTA19, 21 and 25 were identified for direct calving traits. The QTL on BTA19 co-localizes with the reported BH2 haplotype. The QTL on BTA25 is concordant for fertility and calving traits and co-localizes with a QTL previously reported to influence stature and related traits in Brown Swiss dairy cattle.

Conclusion: The detection of QTL and their causative variants remains challenging. Combining comprehensive phenotypic data with imputed whole genome sequences seems promising. We present a QTL on BTA17 for female fertility in dairy cattle with two significantly associated non-synonymous SNPs, along with five additional QTL for fertility traits and calving traits. For all of these we fine mapped the regions and suggest candidate genes and candidate variants.
\end{abstract}

Keywords: Whole genome sequencing, Genome-wide association study, QTL discovery, Functional traits, Brown Swiss, Dairy cattle, Calving ease, Fertility

\footnotetext{
* Correspondence: Mirjam.frischknecht@qualitasag.ch

${ }^{1}$ Qualitas AG, Chamerstrasse 56a, 6300 Zug, Switzerland

${ }^{2}$ School of Agricultural, Forest and Food Sciences HAFL, Bern University of

Applied Sciences, Länggasse 85, 3052 Zollikofen, Switzerland

Full list of author information is available at the end of the article
}

(c) The Author(s). 2017 Open Access This article is distributed under the terms of the Creative Commons Attribution 4.0 International License (http://creativecommons.org/licenses/by/4.0/), which permits unrestricted use, distribution, and reproduction in any medium, provided you give appropriate credit to the original author(s) and the source, provide a link to the Creative Commons license, and indicate if changes were made. The Creative Commons Public Domain Dedication waiver (http://creativecommons.org/publicdomain/zero/1.0/) applies to the data made available in this article, unless otherwise stated. 


\section{Background}

Inadequate fertility and problems associated with calving have high economic importance because they collectively cause $40 \%$ of the involuntary culling that occurs in the Brown Swiss population [1]. Calving ease is also an important because it influences animal welfare. Fertility and calving ease traits are included in routine national evaluations of several countries [2] including the Swiss dairy breeding programs [3, 4]. Genome-wide association studies (GWAS) for fertility and calving ease traits have been performed for several cattle breeds. For female fertility a number of quantitative trait loci (QTL) have been reported in different populations [5-7]. In Danish Jersey cattle the use of imputed whole-genome sequence data allowed the identification of various QTL influencing their national fertility index [8], which includes traits such as number of inseminations per conception, interval from calving to first insemination, 56-day non-return rate and days from first to last insemination. Those QTL were located on bovine chromosome (BTA) 7, 9, 20, 23, 25. Most variants found to have the highest significance of association with that fertility index were intergenic, except one missense variant associated with non-return rate on BTA23. In Italian Holstein, QTL have been identified using 50 K SNP chip data on BTA5, 7, 8, 13, 16, 18 and 27 for days to first service and on BTA2, 17 and 19 for their aggregate fertility index [9].

A locus on BTA18 has been associated with calving ease in Holstein [10]. Other QTL for calving ease have been identified in German Fleckvieh on BTA14 and 21 [5]. The QTL on BTA14 has been associated with stature in German Fleckvieh [11]. In Nordic Red cattle a QTL has been identified to be associated with a sire calving index that includes calving ease, stillbirth rate and a body conformation index including stature [12]. Further genomic associations with calving ease have been found on BTA2 in Limousin and Charolais beef breeds [6]. Overall these studies revealed breed specific loci, often located in regions that are also associated with stature.

Genomic information on thousands of progeny tested bulls is available today as a result of the introduction and now widespread use of genomic selection [13]. With the 1000 Bull Genomes Project [14, 15], a reference panel for imputation to sequence level has become available to project partners. Imputed whole-genome sequence data used in GWAS enhances the discovery of causative variants [16].

The objective of this study was to identify QTL affecting fertility and calving ease traits using imputed wholegenome sequence genotypes of Brown Swiss bulls. Furthermore we aimed to fine-map those QTL to identify potentially causative genes and variants.

\section{Methods \\ Animals}

A total of $\sim 23,000$ Brown Swiss animals genotyped at various densities were available from routine genomic prediction including those involving data sharing among the InterGenomics consortium [17] and the LowInputBreeds project (FP7-project no. KBBE 222623).

\section{Phenotypes}

Estimated breeding values and corresponding reliabilities for five fertility traits were available: non-return rates for heifers (NRH) and cows (NRC) after 56 days; days to first service (DFS); interval between first and last insemination in heifers (IFLH) and in cows (IFLC) [4].

Deregressed breeding values for stillbirth (SB), calving ease (CE), gestation length (GL) and birth weight (BW) were available. For these four calving traits, a GWAS was carried out separately for maternal (m) and for direct (d) effects (Table 1). Additional analyses were undertaken for CEd (calving ease direct) with stature (s) as a covariate (CEd_sc) and for SBd and SBm with exclusion of some of the individuals (SBd corr; SBm corr). In total, breeding values for 13 traits were obtained from the Swiss Brown Swiss routine genomic evaluation [3, 4] and deregressed according to Garrick et al. [18]. We limited the analyses to breeding values of progeny tested bulls with reliabilities of estimated breeding values above 0.55 for fertility traits (except for DFS where the cutoff was 0.65) (Additional file 1: Figure S1) and above 0.20 for calving traits (Additional file 2: Figure S2). These thresholds were chosen to be the same as those used for choosing bulls to be included in the training set for routine genomic prediction. After these filters were applied, there were deregressed breeding values (deregBV) available for GWAS with 1136-4975 bulls depending upon trait (Table 1).

\section{Imputation}

We performed a two-step imputation as this has previously been shown to be more accurate than imputation directly to sequence density [19]. The first step included the imputation from $50 \mathrm{k}$ single nucleotide polymorphism (SNP) chip data to high density (HD) SNP chip data using the software package FImpute with default parameter settings and pedigree information [20]. In the second step we imputed the HD SNPs to sequence density using Minimac with default settings [21] based on sequence variants from the 123 Brown Swiss (BSW) cattle that had been included in run 5 of the 1000 Bull Genomes Project $[14,15]$. Only sequence SNPs and biallelic indels with a minor allele frequency $(\mathrm{MAF})>0.01$ were imputed and only those with R-sq. $>0.1$ in the info file from Minimac were retained for GWAS (Table 1). R-sq. 
Table 1 Minimum, maximum and mean deregressed breeding value (deregBV) and genomic inflation factor lambda and number of individuals included in GWAS per trait

\begin{tabular}{|c|c|c|c|c|c|}
\hline Trait & Min deregBV & Max deregBV & Mean (sd) deregBV & $\begin{array}{l}\text { Genomic inflation } \\
\text { factor (Lambda) }\end{array}$ & Number of individuals \\
\hline $\mathrm{NRH}$ & -75.08 & 64.28 & $0.90(15.68)$ & 0.983 & 2506 \\
\hline NRC & -58.99 & 46.41 & $1.22(14.67)$ & 0.994 & 3615 \\
\hline IFLH & -62.74 & 50.40 & $-0.17(15.34)$ & 0.976 & 1484 \\
\hline IFLC & -52.94 & 46.50 & $-1.39(13.75)$ & 0.990 & 4122 \\
\hline DFS & -61.88 & 41.91 & $-2.69(12.52)$ & 0.990 & 3619 \\
\hline CEd & -210.01 & 121.45 & $-3.37(17.93)$ & 0.974 & 4975 \\
\hline CEd_sc & -210.01 & 121.45 & $-4.32(17.36)$ & 0.992 & 4159 \\
\hline SBd & -114.71 & 291.12 & $-13.00(27.54)$ & 1.010 & 1610 \\
\hline GLd & -121.55 & 156.02 & $0.39(18.26)$ & 0.935 & 2753 \\
\hline BWd & -110.60 & 209.64 & $-0.59(19.69)$ & 0.948 & 2561 \\
\hline CEm & -122.15 & 107.57 & $1.09(25.09)$ & 0.990 & 2862 \\
\hline SBm & -187.92 & 180.70 & $-8.84(28.73)$ & 1.005 & 1136 \\
\hline GLm & -113.34 & 132.43 & $-1.78(23.51)$ & 0.981 & 2756 \\
\hline BWm & -92.46 & 97.23 & $0.82(18.92)$ & 0.978 & 2683 \\
\hline SBd corr & -114.71 & 291.12 & $-14.28(27.80)$ & 1.014 & 1496 \\
\hline SBm corr & -187.92 & 180.70 & $-8.58(29.66)$ & 1.003 & 1051 \\
\hline
\end{tabular}

Trait: $N R H$ non-return rate in heifers, NRC non-return rate in cows, IFLH interval from first to last insemination in heifers, IFLC interval from first to last insemination in cows, DFS Days to first service, CEd calving ease direct, CEd_sc calving ease direct with stature deregressed breeding value (deregBV) as covariate, SBd stillbirth direct, GLd gestation length direct, BWd birth weight direct, CEm calving ease maternal, SBm stillbirth maternal, GLd gestation length maternal, BWd birth weight maternal, SBd corr Stillbirth direct, excluding the smaller cluster, SBm corr, Stillbirth maternal, excluding the smaller cluster SNPs: number of SNPs considered for GWAS after filtering

Min deregBV: Minimum deregressed breeding value for the trait

Max deregBV: Maximum deregressed breeding value for the trait

Mean (sd) deregBV: Mean and standard deviation of the deregressed breeding value for the trait

is an internally calculated value given by Minimac that reflects the imputation quality [21].

\section{Genome-wide association studies}

Genome-wide association studies were conducted using the mixed model approach implemented in EMMAX [22]. We used the $-\mathrm{Z}$ option along with dosage data from imputation as genetic information. Individuals having a pedigree-based gene proportion of Original Braunvieh (OB) [23] > 0.3 were excluded from analysis as animals with high OB-gene proportion would create sub-structures in the population that otherwise mostly comprises individuals with little or no OB-gene proportion. The individual OB-gene proportion and reliability of deregBV were used as covariates in the model. The genomic relationship matrix was calculated for animals included in the analysis for each trait from HD SNP chip genotypes using GCTA [24]. The genomic relationship matrix was used in the mixed model fitted by EMMAX. We performed a principal component analysis (PCA) in $\mathrm{R}$ [25] using the princomp function and plotted the first and the second PC to visualize relatedness captured by the genomic relationship matrix. Using the R package wasim [26] we colored the individuals according to their deregBV. We filtered alleles with $\mathrm{MAF}<0.05$ and those deviating from Hardy-
Weinberg equilibrium (HWE $<1 \times 10^{-20}$ ). After filtering $9,748,130-9,999,287$ variants were included in the GWAS. We used the Bonferroni corrected 0.05 significance threshold and for suggestive threshold we used $p<1 \times 10^{-6}$.

Genomic inflation factor lambda was calculated in $\mathrm{R}$ applying the following formula:

$$
\operatorname{lambda}=\operatorname{round}\left(\operatorname{median}\left(\mathrm{qnorm}(\mathbf{p} / 2)^{\wedge} 2\right) / 0.454,3\right)
$$

where $\mathbf{p}$ is the vector of $p$-values from the EMMAX GWAS.

\section{Variant annotation and description}

All sequence variants were annotated using the Variant effect predictor (VEP) [27]. Frequency estimation of the sequence variants of interest were calculated within and across breeds using data from run 5 data of the 1000 Bull Genomes Project [14]. Linkage disequilibrium between variants was calculated using the -ld funcion in PLINK $[28,29]$. We performed in silico prediction of the impact of missense variants using PolyPHEN2 [30]. Additionally the prediction of the impact of the variant from SIFT was available from VEP. Multiple sequence alignments were done using MAFFT (http://mafft.cbrc.jp/ alignment/server/). 


\section{Results and discussion}

We performed GWAS for five fertility and eight calving traits using filtered imputed whole-genome sequence SNPs assuming that the causative variants were included in the data set. We investigated the QTL we identified for significantly associated variants, in terms of variants with direct impacts on proteins (e.g. missense or frameshift mutations). Most traits we investigated have low heritability (exception GLd with 0.46 ) [3, 4]. We tried to keep our population relatively uniform by excluding animals with an OB-gene proportion $>0.3$. The lambda values indicating genomic inflation (Table 1) reveal that for all traits except SBd and SBm, EMMAX tends to overcorrect for stratification (lambda $<1$ ). In the PCA plot of the genomic relationship matrix for those individuals included for these two traits reveals two clusters (Additional file 3: Figure S3), while for the other traits the PCA plots show that the individuals are uniformly and continuously distributed with respect to the first two principal components (Additional file 3: Figure S3 and Additional file 4: Figure S4). Inspecting the relationships in the smaller cluster of the PCA plot from the individuals included in the GWAS for SB, we found one bull with about 90 male offspring as well as the sire of this bull. This heavily imbalanced relationship is likely to cause the substructure.

\section{Fertility traits}

We found significant associations for each of the five female fertility traits NRH, NRC, IFLH, IFLC and DFS (Fig. 1, Additional file 5: Table S1). The significant QTL were identified on BTA17 (NRH, IFLH and NRC), 25 (IFLC) and 29 (DFS). Beside the three significant associations of the QTL on BTA17, it was also suggestively associated with IFLC. Similarly, the QTL for IFLC on BTA25 was also suggestively associated with DFS. Additionally we found a suggestive QTL for DFS on BTA15.

The QTL on BTA17 is located at around 70-73 Mb. A zoom in this region reveals that the region with the most associated SNPs for IFLC, IFLH and NRH is not significantly associated with NRC (Fig. 2). For that trait, the peak is shifted about $3 \mathrm{Mb}$ and is located around $73 \mathrm{Mb}$ on the same autosome. We speculate that this is a second QTL, which is significant for NRC and NRH. For this QTL, we could identify two missense variants (Table 2). One of the missense variants is significantly associated with NRH and located in the CABIN1 gene. That gene has been found to be significantly associated with fertility traits in Holstein [31] and also differentially expressed in cows from a high and a low fertility group, based on estimated breeding value for calving interval [32]. Even more interesting is the nonsense variant in ENSBTAG00000048030, also located in the $73 \mathrm{Mb}$ QTL. Nonsense variants introduce a premature stop codon and are therefore unlikely to produce a functional protein. Using BLAST we found that the sequence of the corresponding protein has an identity of 84\% with XP_015322696.1, which is encoded by the IGLL1 gene on BTA17. That protein is implicated in immuneresponse and has been shown to be differentially expressed in the endometrium of cows that either showed or did not show signs of estrus around artificial insemination [33]. This gene may play a role in fertilization and therefore we propose the variant in the IGLL1 gene as a candidate variant for fertility traits in dairy cattle. We think that the $73 \mathrm{Mb}$ QTL is present in multiple breeds, such as Italian Holstein [9], while the peak around $71 \mathrm{Mb}$ seems to be specific to the Brown Swiss breed in relation to fertility traits.

There is additional evidence that the genomic regions of these QTL might affect quantitative traits in the Brown Swiss population because two complex copy number variant $(\mathrm{CNV})$ regions have been found to be located at 72-73 $\mathrm{Mb}$ and 73-75 $\mathrm{Mb}$ [34] using the consensus map of $\mathrm{CNV}$ detected from PennCNV and SVS algorithms. A similar region for the Mexican Holstein breed was reported by Duran Aguilar et al. [35] comprising three $\mathrm{CNV}$ regions from $70.7 \mathrm{Mb}$ to

$70.9 \mathrm{Mb}$ mapped using PennCNV. This latter study also reports a significant association with SCS, a functional trait under selection in most of the dairy cattle populations.

The peak region for IFLH is located between $70,462,351$ bp and $71,559,004$ bp on BTA17, which is the center of the $71 \mathrm{Mb}$ QTL. The 70 most associated SNPs of NRH are within this same interval. In this interval two missense variants can be identified to be significantly associated in the GWAS and in the sequenced Brown Swiss animals they are in perfect LD with the top associated variant from the GWAS. The two variants are located in the GAS2L1 gene (g.70,724,328C > T) and in the $A S C C 2$ gene (g.71,084,044G > A).

For both variants the alternative allele has a negative effect on the deregBV. Using in silico effect prediction on the protein, SIFT (provided by VEP) and PolyPHEN2 revealed that the variant in ASCC2 (p.P42L) is likely to have deleterious $(0.02$ - SIFT) or probably damaging (0.992 - PolyPHEN2) impact. The effect of the variant in GAS2L1 (p.P655L) could not reliably be predicted 

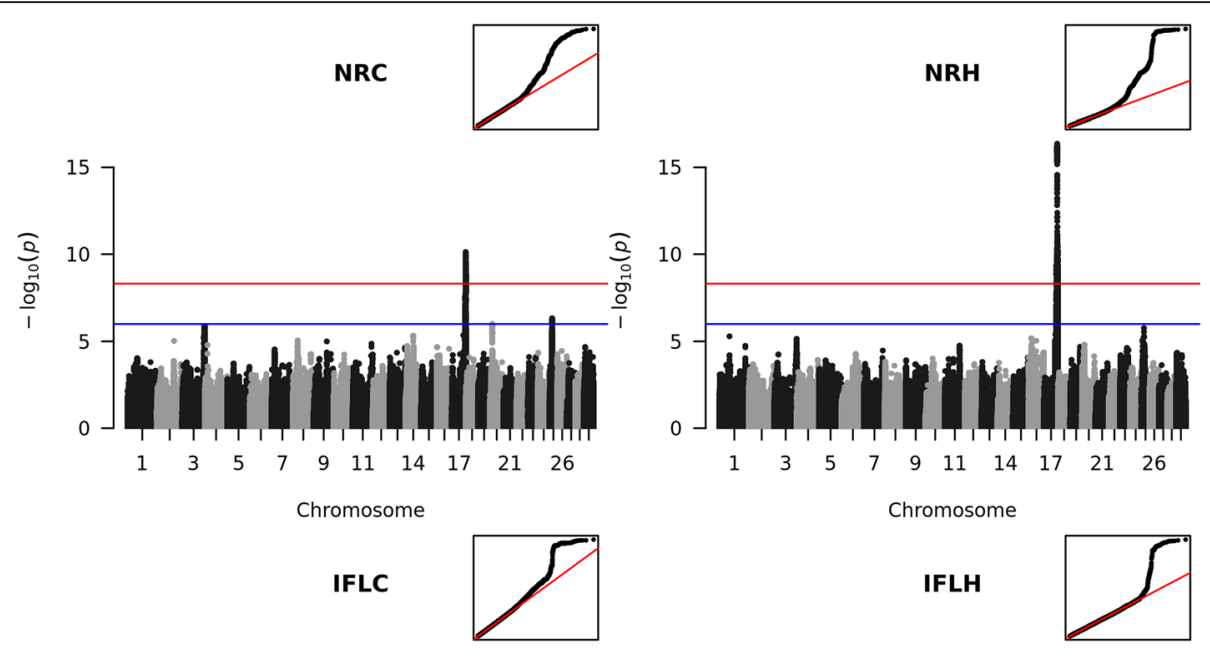

IFLH
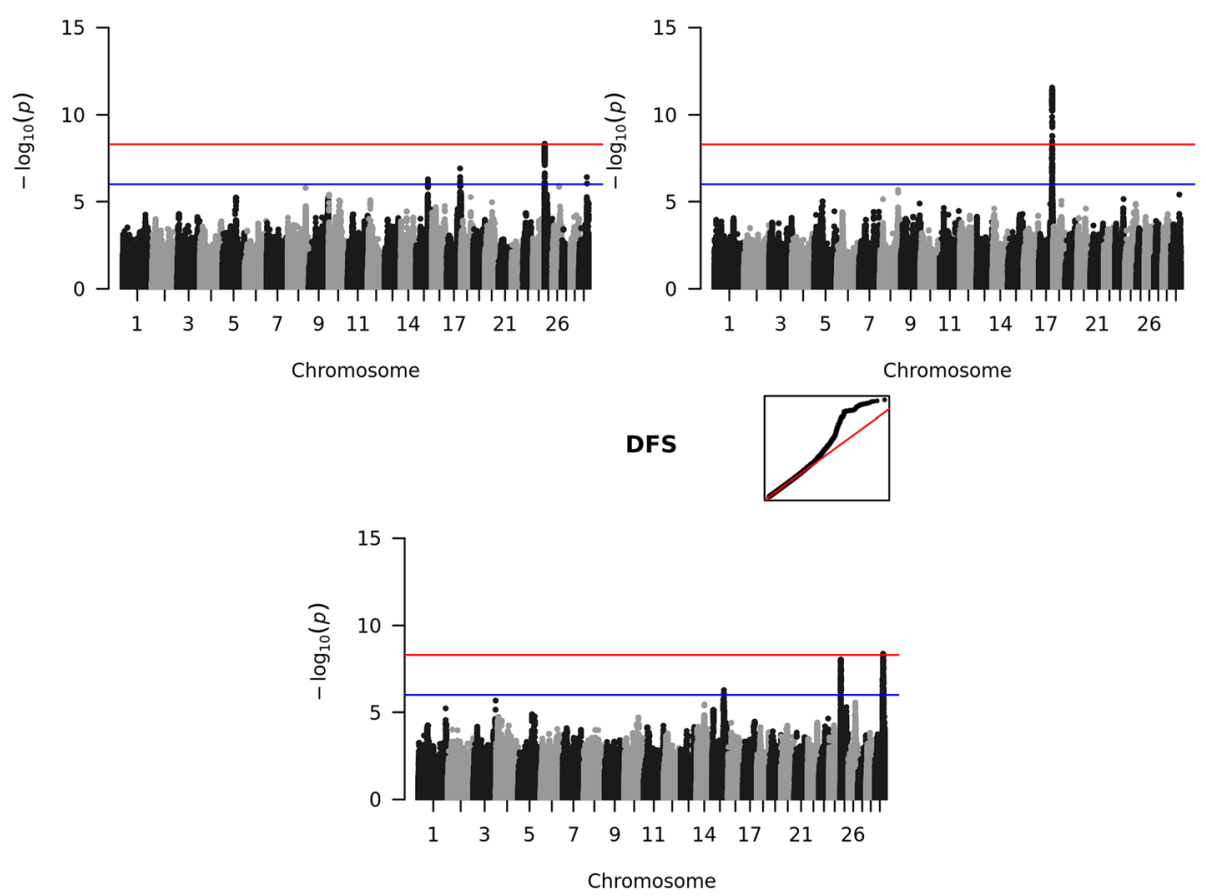

Fig. 1 Manhattan plots for genome-wide association studies for fertility traits. The red line marks the Bonferroni corrected significance threshold. The blue line shows the threshold for suggestive variants. Small figures in the upper right corner show the qqplot of the $p$-values. NRC: Non-return rate in cows; NRH: Non-return rate in heifers; IFLC: Interval from first to last insemination in cows; IFLH: Interval from first to last insemination in heifers; DFS: Days to first service

(deleterious low confidence (0.02); unknown). The variant in $A S C C 2$ is specific to Brown Swiss cattle. In the run 5 of the 1000 Bull Genomes Project, 22 Brown Swiss were found to be heterozygous for this variant whereas 1 animal was homozygous (Additional file 6: Table S2). Additionally we found the variant in two Danish Red cattle, however according to their pedigree, both are sired by animals with breed code BSW. The MAF of this variant is close to $10 \%$ in $\mathrm{BSW}$, while below $1 \%$ across all the sequenced individuals of different breeds. The GAS2L1 p.P655L variant was not only found in BSW but also in Simmental, Angus, Jersey and Hereford. Due to the high LD between these two variants we speculate that the GAS2L1 variant is older and the $A S C C 2$ variant occurred in Brown Swiss on the haplotype carrying the GAS2L1 variant. Since we found homozygous individuals in the data set of sequenced animals for both variants, neither of the variants can be homozygous lethal. ASCC2 is involved in gene activation and repression as part of the ASC1 complex. In order to have support of the hypothesis that the p.P42L variant in ASCC2 is more likely the causative mutation rather than GAS2L1 
NRC

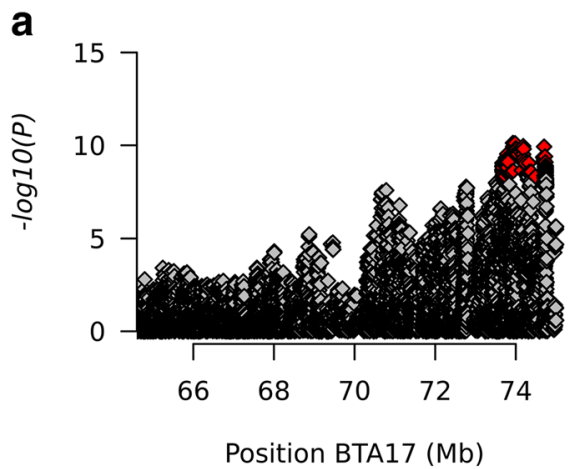

C

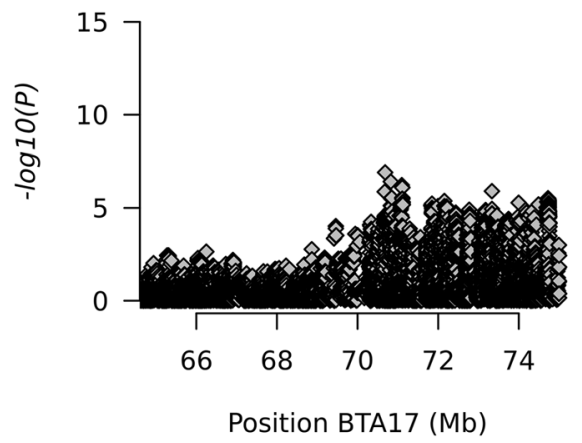

NRH

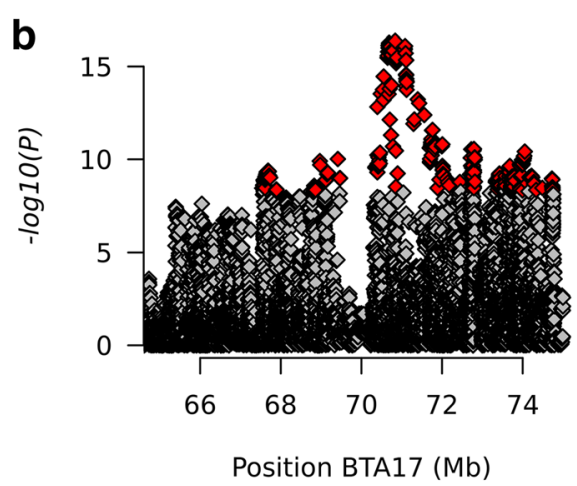

IFLH

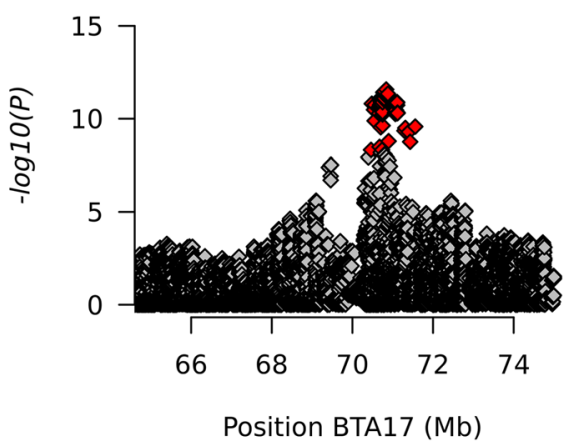

Fig. 2 Association of variants with fertility traits on BTA17 from 66 to $75 \mathrm{Mb}$. The significantly associated variants are marked in red. a Non-return rate in cows (b) Non-return rate in heifers (c) Interval from first to last insemination in cows (d) interval from first to last insemination in heifers

variant. We performed multiple sequence alignments for both p.P42L in ASSC2 and p.P655L in GAS2L1 (Fig. 3). In ASCC2 the amino acid at this position is conserved among all mammals in our comparison and in other species down to the zebra fish. The proline at position 655 in GAS2L1 on the other hand is not conserved at all.

The locus on BTA25 is associated with IFLC. This region on BTA25 has been associated with stature in Brown Swiss cattle [36] and we found it to be associated with calving ease (see below). In Brahman cows it has been shown that larger cattle had lower pregnancy rates [37]. That author suggests the lower pregnancy rate is mainly due to negative energy balance in lactating cows, which cannot be compensated by increased feed intake [37]. This might explain why we do not find this locus to be associated with IFLH, the same fertility trait in heifers, which are not lactating when they become pregnant. For DFS, which is also a trait only measurable in lactating cows, we found a suggestive association for this locus. In a previous study in Brown Swiss this locus has been shown to be associated with a fertility trait (cows ability to recycle after calving) and other traits [36].

The second QTL we identified for a single trait was on BTA29 at around $44 \mathrm{Mb}$ and was associated with DFS. The only significantly associated variant is located in an intron of PYGM. PYGM encodes myophosphorylase, which is a glycogen phosphorylase expressed in muscle. Non-synonymous variants in this gene have been shown to cause protein alterations involved in glycogen storage disease type $\mathrm{V}$ [38]. It remains unclear how this gene could be related to fertility. In the QTL region on BTA29 a second plausible candidate gene is located, which is PLCB3. That gene has been found to be differentially expressed in low and high fertility Holstein cows [32]. Among the significantly associated variants, none were located in this gene. However there is a suggestively associated synonymous variant in PLCB3. Synonymous variants can influence translation efficiency if a rare codon is used [39], which would subsequently influence the level of gene expression and amount of protein available. Since there is differential expression in 
Table 2 Missense and nonsense variants that were significantly $\left(p<5^{*} 10^{-9}\right)$ associated with fertility traits

\begin{tabular}{llllllll}
\hline Trait & BTA & Position & $p$-value & Gene & Variant & PolyPHEN2 & SIFT \\
\hline NRH & 17 & $70,724,328$ & $1.63 \times 10^{-16}$ & GAS2L1 & p.P655L & unknown & deleterious (low confidence) \\
IFLH & & & $2.20 \times 10^{-11}$ & & & & \\
NRH & 17 & $71,084,044$ & $8.03 \times 10^{-17}$ & ASCC2 & p.P42L & probably damaging & deleterious \\
IFLH & & & $3.52 \times 10^{-11}$ & & & & \\
NRH & 17 & $72,747,746$ & $8.78 \times 10^{-10}$ & SLC5A4 & p.G6085 $/ p . G 235 S^{b}$ & probably damaging & deleterious \\
NRH & 17 & $72,815,579$ & $1.61 \times 10^{-9}$ & ENSBTAG00000048030 & p.Y108 & - & - \\
NRH & 17 & $73,344,409$ & $1.39 \times 10^{-9}$ & CABIN1 & p.R651Q & benign & tolerated \\
NRH & 17 & $73,393,194$ & $1.09 \times 10^{-9}$ & CABIN1 & p.A1721V & benign & tolerated (low confidence) \\
NRH & 17 & $73,442,633$ & $2.37 \times 10^{-9}$ & ENSBTAG00000046900 & p.T234A & benign & tolerated \\
NRC & 17 & $74,739,013$ & $3.25 \times 10^{-9}$ & CDC45L & p.A263T & benign & tolerated \\
NRH & & & $3.49 \times 10^{-9}$ & & & &
\end{tabular}

Trait: $N R C$ non-return rate in heifers, $N R H$ non-return rate in heifers, IFLH interval from first to last insemination in heifers

BTA: Chromosome

$P$-value: From GWAS with EMMAX

Variant: Amino acid change caused by the variant

PolyPhen2: Predicted effect of the variant on the protein function from PolyPhen2

SIFT: Predicted effect of the variant on the protein function from SIFT obtained via Variant effect predictor (VEP)

${ }^{\mathrm{a} / \mathrm{b}}$ The transcripts ENSBTAT00000010678 ${ }^{\mathrm{a}}$ and ENSBTAT00000052915 ${ }^{\mathrm{b}}$ for SLC5A4

low and high fertility Holstein cows a lower translation rate and a subsequent lower amount of protein for PLCB3 could potentially impact fertility.

\section{Calving traits}

For the direct calving traits we could identify significantly associated regions for the three traits CEd, SBd and GLd (Fig. 4, left side, Additional file 5: Table S1). Only for BWd no locus was found to be significantly associated with the trait. Unlike for the fertility traits, we found different QTL for each calving trait. Those QTL with significant association were located on BTA19, 21 and 25. Additionally we identified suggestive QTL on BTA5, 22 and 29.

\section{Direct calving ease and gestation length}

For direct calving ease (CEd) two QTL were identified, on the proximal ends of BTA21 and BTA25. The locus on BTA25 has been associated with stature in Brown Swiss cattle [36]. Intuitively, it seems logical that large calves would determine more birth difficulties than small calves when born to cows of the same stature. However

\section{a Bos_taurus Homo_sapiens \\ Pan_troglodytes \\ Mus_musculus \\ Rattus_norvegicus Xenopus_tropicalis Danio_rerio}

b
FVLYKPPPKDNIPAL

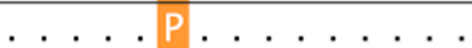

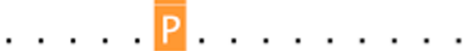

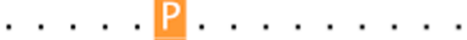

$\ldots . . . . . .$.

...VP. . ASCA...

. LPF.P. . E.GT...

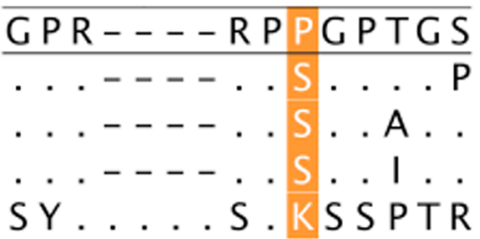

Fig. 3 Multiple sequence alignment of (a) ASCC2 (amino acids 37-51 (Bos taurus)) the orange shading marks the p.42 position. The sequences for ASCC2 were derived from the following accession numbers: Bos taurus, NP001015524.1; Homo sapiens, NP_115580.2; Pan troglotydes, XP_515064.3; Mus musculus, NP_083567.1; Rattus norvegicus, NP_001102561.1; Xenopus tropicalis, NP_001016871.1; Danio rerio, NP_956736.1 and (b) GAS2L1 (amino acids 650- the orange shading marks the p.655 position. The sequences for GAS2L1 were derived from the following accession numbers: Bos taurus, NP_001077167.1; Canis lupus familiaris, XP_543468.2; Homo sapiens, NP Mus musculus, NP_653146.1; Xenopus tropicalis, XP_002934334.1 

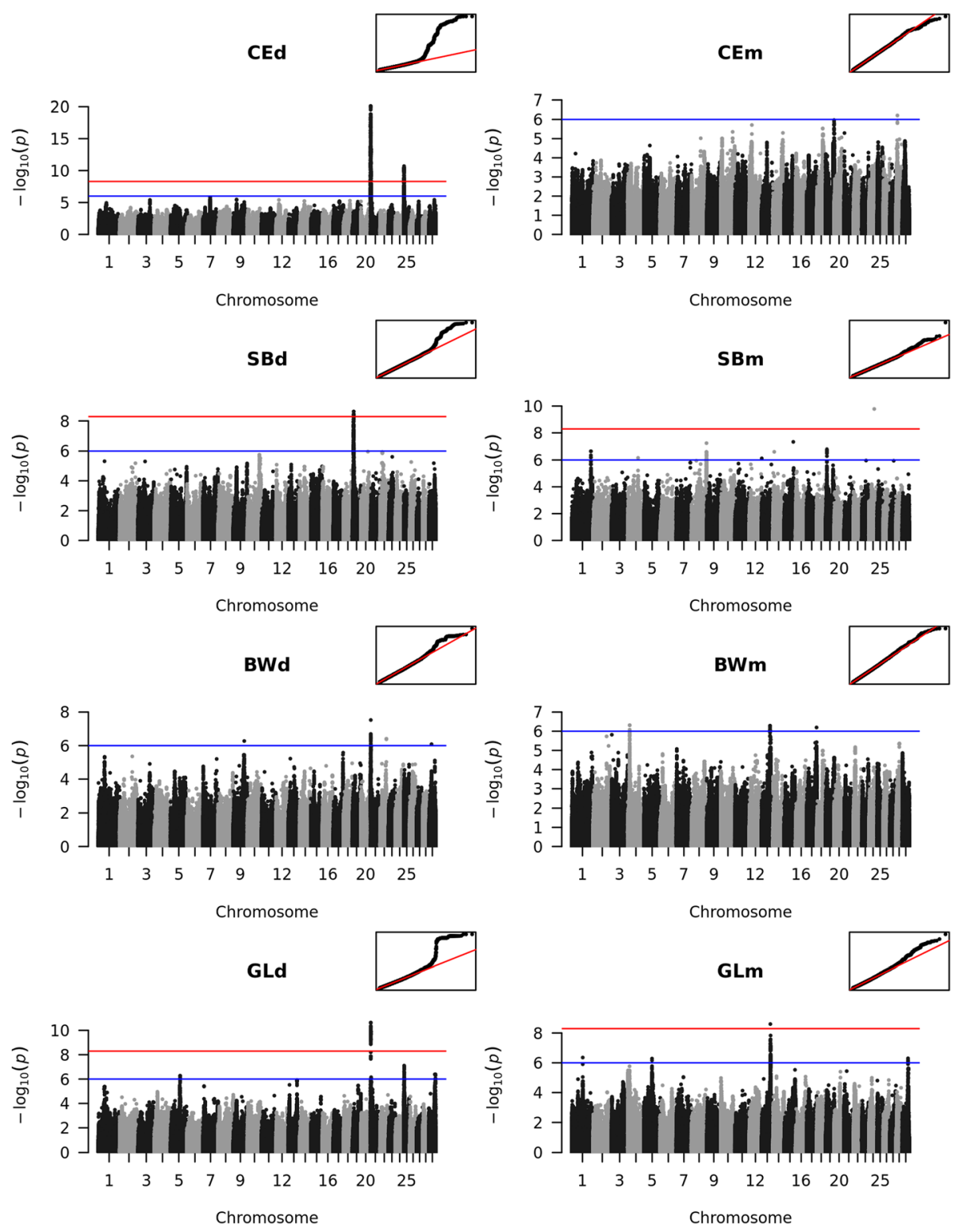

Fig. 4 Manhattan plots for the genome-wide association studies for calving traits. The red line marks the Bonferroni corrected significance threshold. The blue line shows the threshold for suggestive variants. Small figures in the upper right corner show the corresponding qqplot. CEd: Calving ease direct; CEm: Calving ease maternal; SBd: Stillbirth direct; SBm: Stillbirth maternal; BWd: Birth weight direct BWm: Birth weight maternal; GLd: Gestation length direct; GLm: Gestation length maternal

genetic correlations between stature and calving ease are non-significant in UK Holstein-Friesian cattle [40]. We also found only a low correlation between those two traits of 0.1 for the phenotypic correlation of the deregressed breeding values of the two traits. This might be explained by the fact that not all loci influencing adult size have an impact on the size of the calf. However, we additionally performed GWAS for calving ease using the deregressed breeding value for stature as a covariate. With stature as a covariate the signal on BTA25 disappears. (Additional file 7: Figure S5). We interpret, that the locus on BTA25 is either pleiotropic or that the phenotypes observed are indeed due to differences in the size of the calves, with small calves being delivered more easily than tall calves. Even though this QTL has been described previously, no possible causative variant has been identified. For CEd, we found three missense variants among the significantly associated variants on BTA25, located in CRAMP1L, PTX4 and TELO2 genes (Table 3). All three variants were significantly associated with stature in BSW as well as CEd. The direction of the SNP effects was however opposite. According to 
Table 3 Missense and nonsense variants that were significantly $\left(p<5^{*} 10^{-9}\right)$ associated with calving traits

\begin{tabular}{llllllll}
\hline Trait & BTA & Position & $p$-value & Gene & Variant & PolyPHEN2 & SIFT \\
\hline CEd & 25 & $1,277,577$ & $5.59 \times 10^{-10}$ & CRAMP1L & p.R885Q & probably damaging \\
CEd & 25 & $1,177,995$ & $1.93 \times 10^{-9}$ & TELO2 & p.R536W & probably damaging & deleterious \\
CEd & 25 & $1,161,904$ & $4.05 \times 10^{-9}$ & PTX4 & p.P207L & probably damaging & tolerated \\
\hline
\end{tabular}

Trait: CEd calving ease direct

BTA: Chromosome

$P$-value: From GWAS with EMMAX

Variant: Amino acid change caused by the variant

PolyPhen2: predicted effect of the variant on the protein function from PolyPhen2

SIFT: predicted effect of the variant on the protein function from SIFT obtained via Variant effect predictor (VEP)

* : Translation stop codon

PolyPHEN2 all three variants are probably damaging and therefore potentially causal. As for regions on BTA17, the CNVR_510 reported by Prinsen et al. [34] on BTA25 in BSW spans these significantly associated missense variants. The genomic variation CNVR_510 is detected in 86 individuals indicating that the $\mathrm{CNV}$ in this location may represent an important source of variation that may affect quantitative traits.

The locus on BTA21 remained significantly associated with calving ease when including stature as a covariate (Additional file 7: Figure S5). Therefore this locus is likely not involved in normal variation in adult stature. Interestingly the same locus had been identified earlier in German Fleckvieh cattle to be associated with the complex of calving traits [5]. However in that study an association of SB with the same QTL was reported, whereas we could not detect any association with SB in Brown Swiss. Moreover, we found this region to be associated with gestation length. Generally a longer gestation length is associated with higher birth weight and a subsequent increase in calving difficulties [3].

The direction of the SNP effects within the QTL on BTA 21 highlight this relationship between gestation length and calving ease. The mechanism by which this region could impact calving ease is unknown. It is known that the shared syntenic region in human is imprinted and associated with Prader-Willi syndrome for defects in the paternal allele and with Angelman syndrome for the maternal allele [41]. Human babies with Prader-Willi syndrome have an increased risk of being born by cesarean section [42], which would be in agreement with a decreased breeding value for calving ease. However on the other hand a further feature of PraderWilli syndrome is that offspring are at increased risk of preterm birth, which does not match the expectation and known relationship between GL and CE.

We found 7 synonymous variants significantly associated with ATP10A. The ATP1OA gene is maternally imprinted in human and associated with Angelman syndrome [43]. However, there is some evidence that this gene is not imprinted in mice [44]. We found suggestively associated non-synonymous variants in ATP10A and MAGEL2. The one located in the ATP10A gene, causes p.M655 L, predicted by SIFT to be tolerated and by PolyPHEN2 to be benign. The other variant causes an amino acid exchange from Glutamic acid to Glycine at the 851 amino acid position. For this variant no prediction about its effect size could be done. MAGEL2 is implicated in Prader-Willi syndrome [45], which phenotypically seems to have a larger influence on birth traits than Angelman syndrome. For MAGEL2 in bovine parthenogenic and normal embryonic cells no difference in the expression pattern could be detected suggesting this gene is not imprinted in cattle [46]. For mice that are heterozygous for MAGEL2 deficiency, an increased perinatal lethality has been described [47].

For GLd, the top associated variants are about $800 \mathrm{~kb}$ downstream compared to those for CEd. The SNPs in this region are also significantly associated with CEd. When analysing the SNP density in this region we found that there is a decreased SNP density at the beginning of BTA21 (less than 1000 SNPs per Mb) relative to other locations. This observation is displayed in the zoomed plot (Additional file 8: Figure S6). The SNP density is similar for both traits in this region. We speculate that the causative variant is indeed the same for both traits.

\section{Stillbirth}

We identified several SNPs on BTA19 with significant associations with $\mathrm{SB}$ at around $10 \mathrm{Mb}$. That locus colocalizes with the $\mathrm{BH} 2$ haplotype for which a causative mutation has been identified in the TUBD1 gene [48]. Calves homozygous for the TUBD1 variant show high mortality rates during or shortly after birth. In our data set the MAF of this variant was lower than 0.05 and therefore it was not included in the GWAS, and in any event the imputation R-sq. was rather low (0.12) for this variant. However, because this variant is an obvious candidate causative variant for this gene we ran an additional single SNP regression for this variant generating a non-significant $p$-value of $4.68 \times 10^{-4}$. Interestingly having a closer look at the $p$-values in the BH2 haplotype region, there were reduced $-\log _{10}$ ( $\mathrm{p}$-values) observed compared to slightly upstream and 


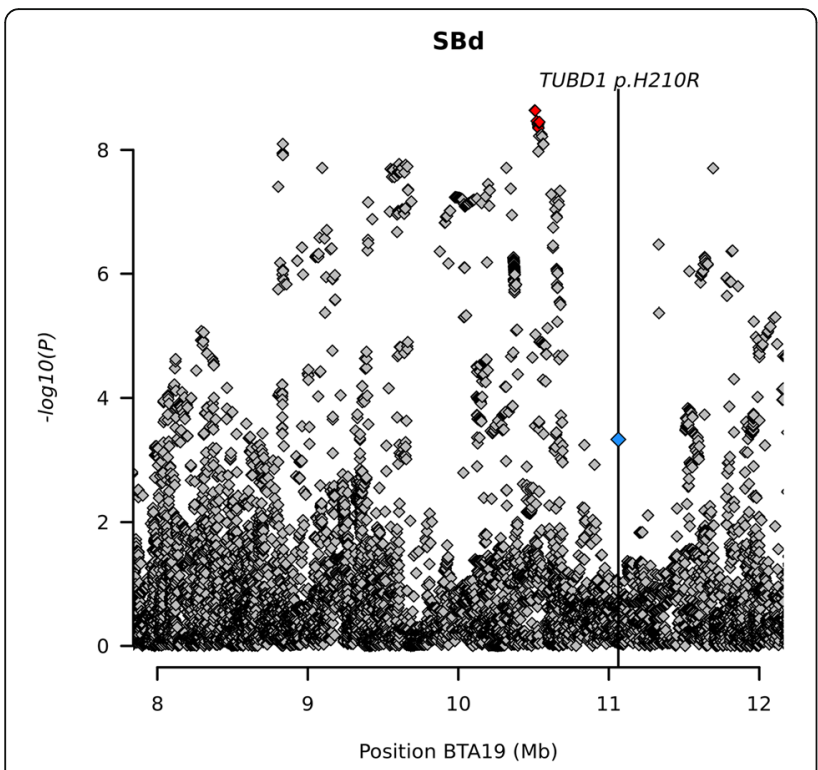

Fig. 5 Association of stillbirth (direct) on BTA19 and location of TUBD1 variant causing the $\mathrm{BH} 2$ phenotype. The significantly associated variants are marked in red

downstream from the haplotype (Fig. 5). We have to remember that the model assumption for which association testing is performed is an additive mode of inheritance, but $\mathrm{BH} 2$ is never present in homozygous form. It is possible that another QTL is responsible for the signal. In order to verify that the association is truly caused by $\mathrm{BH} 2$, we removed $\mathrm{BH} 2$ carriers in an additional GWAS and in a second step we used $\mathrm{BH} 2$ status as a covariate. In both cases no significant SNP remained on BTA19. Therefore we conclude that the signal is indeed caused by BH2 (Additional file 9: Figure S7).

\section{Maternal traits}

We also performed GWAS for the maternal calving traits CEm, SBm, BWm and GLm (Fig. 4, right side, Additional file 5: Table S1). We found one SNP significantly associated with SBm. That SNP has an imputation R-sq. of 0.17 and MAF of 0.051 . These values are only slightly above the quality control thresholds and therefore we believe that the significant effect of this SNP may represent a false positive. This is corroborated by the fact that no other SNP in that region shows either significant or suggestive association. There is a substructure in the individuals used for SBm and we believe this false-positive association may be due to that (Additional file 3: Figure S3 C and D). To support this hypothesis, we reran the GWAS excluding all individuals in the smaller cluster of the PCA plot (the sire with 90 offspring, its father and its siblings). The Manhattan plot clearly demonstrates that there is no longer any significantly associated SNP (Additional file 10: Figure S8).
Because a reduction in the number of individuals also leads to a reduction of power to detect true associations, we removed the same individuals for SBd, and there the peak on BTA19 remained significant. These results show how important a good correction and an eventual exclusion of outlier individuals is in order to avoid falsepositive findings.

The other maternal trait for which we found a significant association was GL, the association for GLm is only due to a single significant SNP but a number of nearby SNPs are suggestively associated, just below the significance threshold. Additionally this SNP has a higher imputation R-sq. (0.781) and MAF (0.383). The QTL for GLm was located on BTA13. Zooming on this region reveals that there could actually be 2 QTL: at $65.5 \mathrm{Mb}$ with the significant SNP and a suggestive QTL at 67.1 $\mathrm{Mb}$ (Fig. 6). The two loci are not in strong LD $\left(\mathrm{r}^{2}=0.35\right)$, supporting the hypothesis of two QTL in this region. The significantly associated variant at around $65.5 \mathrm{Mb}$, is located in an intron of CPNE1. CPNE1 encodes a calcium-dependent phospholipid binding protein but little is known about the exact role of the protein. Recently it has been found that this gene is expressed in human placenta [49] but not found to be expressed above 50 fragments per kilobase million (FPKM) in the cattle transcriptome [49]. However the placentas used in that study came from term births and therefore differential expression over gestation cannot be ruled out. Totally 142 suggestively associated variants are distributed on the two QTL. Of these 15 are associated with the first QTL and include a missense variant in the SPAG4 gene. The variant is p.Y289F and is predicted to be tolerated

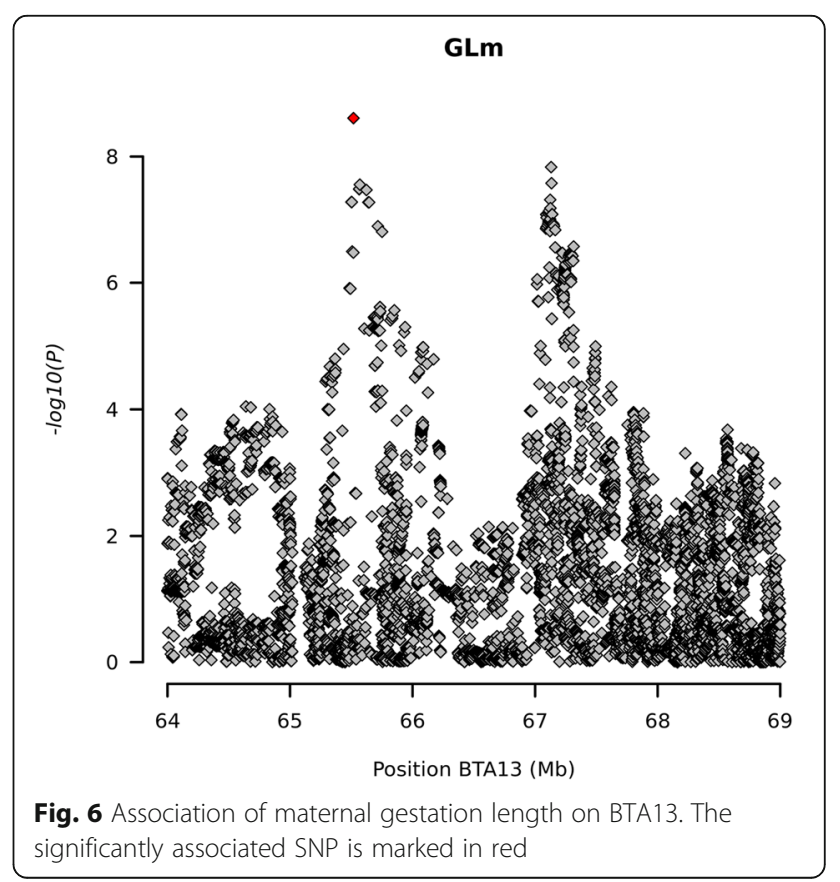


by SIFT, but PolyPHEN2 predicts that the variant is probably damaging. SPAG4 encodes the spermassociated antigen protein 4 . As the name indicates, disruption of this gene leads to abnormal sperm development and decreased male fertility [50]. Additionally this gene has also been found to be differentially expressed in pre-eclamptic placenta in humans [51]. This gene is an interesting candidate gene for GLm.

At the second locus 127 variants are suggestively associated. Among these variants we find a single synonymous variant in the CTNNBL1 gene. This gene has been found to be expressed in the placenta of Ateles fusciceps but not with FPKM $>50$ in cattle. Deficiency of CTNNBL1 in mice leads to embryonic lethality [52]. More interestingly for GLm are the genes NNAT and $B L C A P$. The peak of this second QTL is located between these two genes. Both genes are imprinted and expressed in the placenta [53]. Therefore these two genes provide good candidate genes for a maternal trait. We cannot suggest a possible causative variant. Variants with influence on gene expression but with no alteration of the protein are hard to detect. It is likely that the causative variant is located outside the coding region.

\section{Conclusion}

In this study we used readily available phenotypes that are collected for routine genomic prediction to identify QTL for traits related to fertility and calving ease. We detected a novel QTL for fertility in Brown Swiss on BTA17 and suggest a candidate that may represent the causal variant. We further identified regions associated with other birth and fertility traits. We detected a region with a known deleterious variant to be significantly associated with stillbirth and a region associated with maternal gestation length including genes with placental expression in that associated region. This study gives insight into the genetic architecture of the functional traits that characterize fertility and calving ease.

\section{Additional files}

Additional file 1: Figure S1. Distribution of reliabilities of estimated breeding values for fertility traits. NRC: Non-return rate in cows; NRH: Non-return rate in heifers; IFLC: Interval from first to last insemination in cows; IFLH: Interval from first to last insemination in heifers; DFS: Days to first service. (PNG $51 \mathrm{~kb}$ )

Additional file 2: Figure S2. Distribution of reliabilities of estimated breeding values for calving traits. CEd: Calving ease direct; CEm: Calving ease maternal; SBd: Stillbirth direct; SBm: Stillbirth maternal; BWd: Birth weight direct BWm: Birth weight maternal; GLd: Gestation length direct; GLm: Gestation length maternal. (PNG 57 kb)

Additional file 3: Figure S3. The first two principal coponents for the genomic relationship matrix comprising individuals used for genome-wide association of calving traits (A) Calving ease direct (B) Calving ease maternal (C) Stillbirth direct (D) Stillbirth maternal (E) Birth weight direct (F) Birth weight maternal (G) Gestation length direct (H) Gestation length maternal. (PNG 816 kb)

Additional file 4: Figure S4. The first two principal coponents for the genomic relationship matrix comprising individuals used for genome-wide association of fertility traits (A) Non-return rate in cows (B) Non-return rate in heifers (C) Interval from first to last insemination in cows (D) Interval from first to last insemination in heifers (E) Days to first service. (PNG $677 \mathrm{~kb}$ )

Additional file 5: Table S1. GWAS results per trait. SNPs with $p$-values $<10^{-6}$ for each trait. (XLSX $415 \mathrm{~kb}$ )

Additional file 6: Table S2. Minor allele frequency per breed among sequenced individuals for ASCC2 and GAS2L1. (XLSX $44 \mathrm{~kb}$ )

Additional file 7: Figure S5. GWAS for calving ease using stature as a covariate. The red line marks the Bonferroni corrected significance threshold. The blue line shows the threshold for suggestive variants. The figure in the upper right corner shows the corresponding qqplot. (PNG $74 \mathrm{~kb}$ )

Additional file 8: Figure S6. Association for calving ease direct (CEd) and gestation length direct (GLd) on BTA21 from 1 to $4 \mathrm{Mb}$. The red line indicates the Bonferroni corrected significance threshold. (PNG $582 \mathrm{~kb}$ )

Additional file 9: Figure S7. Association on BTA19 for stillbirth using only individuals not carrying $\mathrm{BH} 2$ (top) or using $\mathrm{BH} 2$ carrier status as a covariate (bottom). The red line indicates the Bonferroni corrected significance threshold. (PNG $563 \mathrm{~kb}$ )

Additional file 10: Figure S8. Manhattan plots for the GWAS for stillbirth direct and maternal excluding individuals from the smaller cluster in the PCA plot. The red lines mark the Bonferroni corrected significance threshold. The blue lines show the threshold for suggestive variants. Small figures in the upper right corner shows the corresponding qqplot. (PNG $506 \mathrm{~kb}$ )

\section{Abbreviations}

BSW: Brown Swiss Cattle; BTA: Bovine chromosome; BWd: Birth weight direct; BWm: Birth weight maternal; CEd: Calving ease direct; CEm: Calving ease maternal; CNV: Copy number variant; deregBV: Deregressed breeding value; DFS: Days to first service; FPKM: Fragments per kilobase million; GLd: Gestation length direct; GLm: Gestation length maternal; GWAS: Genome-wide association study; HD: High density; IFLC: Interval from first to last insemination in cows; IFLH: Interval from first to last insemination in heifer; MAF: Minor allele frequency; NRC: Non-return rate in cows; NRH: Non-return rate in heifers; PCA: Principal component analysis; QTL: Quantitative trait locus/loci; SBd: Stillbirth direct; SBm: Stillbirth maternal; SNP: Single nucleotide polymorphism; VEP: Variant effect predictor

\section{Acknowledgements}

We thank the 1000 Bull Genomes Project for providing the sequencing data, which has been used as a reference for the imputation and for the estimation of minor allele frequencies. We acknowledge Alessandro Bagnato for valuable comments on the manuscript. Furthermore, we thank the Fondation sur la croix and the Swiss Commission for Technology and innovation for financial support of this project.

\section{Funding}

This study was partly conducted within the Swiss Low Input Genetics (SLIG) project, which was financially supported by the Swiss Commission for Technology and Innovation, and partly funded by the Fondation sur la Croix.

\section{Availability of data and materials}

All relevant data are included in the body of the manuscript and its additional files. Sequence data of all animals are part of the run 5 of the 1000 Bull Genomes Project (http://www.1000bullgenomes.com). The variants found in Brown Swiss cattle have been reported to dbSNP (https:/www.ncbi.nlm.nih.gov/snp/). The raw data belong to more than 10 institutions and breeding organizations. The data is commercially sensitive and cannot be made publicly available. Contact details of representatives of the partners are available upon request from the corresponding author. 


\section{Authors' contributions}

$\mathrm{MF}, \mathrm{BB}, \mathrm{FRS}, \mathrm{CF}, \mathrm{BG}$ conceived the study, carried out all analyses and wrote the manuscript. HSH, DG, CS, RF, IR, IC, IR, JS, AB and MGS contributed data and helped in the analyses. All authors read and approved the final manuscript.

\section{Ethics approval}

No experimental animal studies were conducted for the work in this manuscript. The sequence data for all animals were obtained from the 1000 bulls genome project [14]. The deregressed breeding values were obtained from routine genomic prediction.

\section{Consent for publication}

Not applicable.

\section{Competing interests}

The authors declare that they have no competing interests.

\section{Publisher's Note}

Springer Nature remains neutral with regard to jurisdictional claims in published maps and institutional affiliations.

\section{Author details \\ 'Qualitas AG, Chamerstrasse 56a, 6300 Zug, Switzerland. ${ }^{2}$ School of Agricultural, Forest and Food Sciences HAFL, Bern University of Applied Sciences, Länggasse 85, 3052 Zollikofen, Switzerland. ${ }^{3}$ Institute of Veterinary, Animal \& Biomedical Sciences, Massey University, Hamilton, New Zealand. ${ }^{4}$ agn Genetics GmbH, 8b Börtjistrasse, 7260 Davos, Switzerland. ${ }^{5}$ Interbull center, SLU - Box 7023, S-75007 Uppsala, Sweden. ${ }^{6}$ Technische Universität München, Liesel-Beckmann-Straße 1, 85354 Freising-Weihenstephan, Germany. ${ }^{7}$ Tierzuchtforschung e.V, Senator-Gerauer-Str. 23, 85586 Poing, Germany. ${ }^{8}$ University of Natural Resources and Life Sciences, Gregor-Mendel-Str 33, 1180 Wien, Austria. ${ }^{9}$ Research Institute of Organic Agriculture (FiBL), Ackerstrasse 113, 5070 Frick, Switzerland. ${ }^{10}$ Department of Veterinary Medicine, University of Milan, Via Celoria 10, 20133 Milan, Italy.}

\section{Received: 14 August 2017 Accepted: 15 November 2017}

\section{Published online: 25 November 2017}

\section{References}

1. Burren A, Alder S. Abgangsursachen und LBE. CHbraunvieh. 2013:3:8-11.

2. Egger-Danner C, Cole JB, Pryce JE, Gengler N, Heringstad B, Bradley A, et al. Invited review: overview of new traits and phenotyping strategies in dairy cattle with a focus on functional traits. Animal. 2015;9:191-207.

3. Berweger M. Nur noch TVD-Daten für die ZWS Geburtsablauf. CHbraunvieh. 2016:4:10-2

4. Gredler B, Schnyder U. New genetic evaluation of fertility in Swiss Brown Swiss. Interbull Bull. 2013:47:226-9.

5. Pausch H, Flisikowski K, Jung S, Emmerling R, Edel C, Götz KU, et al. Genome-wide association study identifies two major loci affecting calving ease and growth-related traits in cattle. Genetics. 2011;187:289-97.

6. Purfield DC, Bradley DG, Evans RD, Kearney FJ, Berry DP. Genome-wide association study for calving performance using high-density genotypes in dairy and beef cattle. Genet Sel Evol. 2015;47:47

7. Schulman NF, Sahana G, Iso-Touru T, McKay SD, Schnabel RD, Lund MS, et al. Mapping of fertility traits in Finnish Ayrshire by genome-wide association analysis. Anim Genet. 2011;42:263-9.

8. Höglund JK, Guldbrandtsen B, Lund MS, Sahana G. Identification of genomic regions associated with female fertility in Danish Jersey using whole genome sequence data. BMC Genet. 2015;16:60

9. Minozzi G, Nicolazzi EL, Stella A, Biffani S, Negrini R, Lazzari B, et al. Genome wide analysis of fertility and production traits in Italian Holstein cattle. PLoS One. 2013:8:e80219.

10. Müller M-P, Rothammer S, Seichter D, Russ I, Hinrichs D, Tetens J, et al. Genome-wide mapping of 10 calving and fertility traits in Holstein dairy cattle with special regard to chromosome 18. J Dairy Sci. 2017:100:1-20.

11. Pausch $H$, Emmerling $R$, Schwarzenbacher $H$, Fries R. A multi-trait meta-analysis with imputed sequence variants reveals twelve QTL for mammary gland morphology in Fleckvieh cattle. Genet Sel Evol. 2016;48:14.

12. Sahana G, Höglund JK, Guldbrandtsen B, Lund MS. Loci associated with adult stature also affect calf birth survival in cattle. BMC Genet. 2015;16:47.
13. Meuwissen THE, Hayes BJ, Goddard ME. Prediction of total genetic value using genome-wide dense marker maps. Genetics. 2001;157:1819-29.

14. Daetwyler HD, Capitan A, Pausch H, Stothard P, van Binsbergen R, Brøndum RF, et al. Whole-genome sequencing of 234 bulls facilitates mapping of monogenic and complex traits in cattle. Nat Genet. 2014;46:858-65.

15. 1000 bull genomes project. Available from: http://www.1000bullgenomes.com/.

16. Pausch H, Macleod IM, Fries R, Emmerling R, Phil J. Evaluation of the accuracy of imputed sequence variants and their utility for causal variant detection in cattle. Genet Sel Evol. 2017:49:24

17. Jorjani H, Jakobsen J, Nilforooshan MA, Hjerpe E, Zumbach B, Palucci V. Genomic evaluation of BSW populations InterGenomics: results and deliverables. Interbull Bull. 2011:43:5-8.

18. Garrick DJ, Taylor JF, Fernando RL. Deregressing estimated breeding values and weighting information for genomic regression analyses. Genet Sel Evol. 2009;41:55.

19. Khatkar MS, Moser G, Hayes BJ, Raadsma HW. Strategies and utility of imputed SNP genotypes for genomic analysis in dairy cattle. BMC Genomics. 2012;13:538

20. Sargolzaei M, Chesnais J, Schenkel F. Flmpute - an efficient imputation algorithm for dairy cattle populations. J Anim Sci/J Dairy Sci. 2011;89/94:421.

21. Fuchsberger $C$, Abecasis GR, Hinds DA. Minimac2: faster genotype imputation. Bioinformatics. 2014;31:782-4.

22. Kang HM, Sul JH, Service SK, Zaitlen NA, Kong S-Y, Freimer NB, et al. Variance component model to account for sample structure in genome-wide association studies. Nat Genet. 2010;42:348-54.

23. Flury C, Boschun C, Denzle M, Baps B, Schnyde U, Gredle B, et al. Genomewide association study for 13 udder traits from linear type classification in cattle. Proc. 10th world Congr. Genet. Appl. To Livest. Prod. 2014

24. Yang J, Lee SH, Goddard ME, Visscher PMGCTA. A tool for genome-wide complex trait analysis. Am J Hum Genet. 2011:88:76-82.

25. R Core Team. A language and environment for statistical computing http://www.R-project.org/.

26. Reusser D, Francke T. wasim: Visualisation and analysis of output files of the hydrological model WASIM. 2011. Available from: https://cran.r-project.org/ package $=$ wasim

27. McLaren W, Gil L, Hunt SE, Riat HS, Ritchie GRS, Thormann A, et al. The Ensembl variant effect predictor. Genome Biol. 2016;17:122.

28. Chang CC, Chow CC, Tellier LC, Vattikuti S, Purcell SM, Lee JJ. Second-generation PLINK: rising to the challenge of larger and richer datasets. Gigascience. 2015;4

29. Purcell S, Neale B, Todd-Brown K, Thomas L, Ferreira MAR, Bender D, et al. PLINK: a tool set for whole-genome association and population-based linkage analyses. Am J Hum Genet. 2007:81:559-75.

30. Adzhubei IA, Schmidt S, Peshkin L, Ramensky VE, Gerasimova A, Bork P, et al. A method and server for predicting damaging missense mutations. Nat Methods. 2010:7:248-9.

31. Höglund JK, Sahana G, Guldbrandtsen B, Lund MS. Validation of associations for female fertility traits in Nordic Holstein, Nordic red and Jersey dairy cattle. BMC Genet. 2014;15:8

32. Moore SG, Pryce JE, Hayes BJ, Chamberlain AJ, Kemper KE, Berry DP, et al. Differentially expressed genes in Endometrium and corpus Luteum of Holstein cows selected for high and low fertility are enriched for sequence variants associated with fertility. Biol Reprod. 2016;94:19.

33. Davoodi S, Cooke RF, Fernandes ACC, Cappellozza BI, Vasconcelos JLM, Cerri RLA. Expression of estrus modifies the gene expression profile in reproductive tissues on day 19 of gestation in beef cows. Theriogenology. 2016;85:645-55

34. Prinsen RTMM, Strillacci MG, Schiavini F, Santus E, Rossoni A, Maurer V, et al. A genome-wide scan of copy number variants using high-density SNPs in Brown Swiss dairy cattle. Livest Sci. 2016;191:153-60.

35. Duran Aguilar M, Roman Ponce SI, Ruiz Lopez FJ, Gonzalez Padilla E, Vasquez Pelaez CG, Bagnato A, et al. Genome-wide association study for milk somatic cell score in holstein cattle using copy number variation as markers. J Anim Breed Genet. 2017;134:49-59.

36. Guo J, Jorjani H, Carlborg Ö. A genome-wide association study using international breeding-evaluation data identifies major loci affecting production traits and stature in the Brown Swiss cattle breed. BMC Genet. 2012;13:82.

37. Olson TA. Reproductive efficiency of cows of different sizes. 1993. Available from: http://animal.ifas.ufl.edu/beef_extension/bcsc/1993/docs/olson.pdf

38. Martín MA, Lucía A, Arenas J, et al. Glycogen Storage Disease Type V. In: Adam MP, Ardinger HH, Pagon RA, et al, editors. GeneReviews ${ }^{\oplus}$. Seattle: 
University of Washington, Seattle; 2006. pp. 1993-2017. Available from: https://www.ncbi.nlm.nih.gov/books/NBK1344/.

39. Quax TEF, Claassens NJ, Söll D, van der Oost J. Codon bias as a means to fine-tune gene expression. Mol Cell. 2015;59:149-61.

40 Eaglen SAE, Coffey MP, Woolliams JA, Wall E. Direct and maternal genetic relationships between calving ease, gestation length, milk production, fertility, type, and lifespan of Holstein-Friesian primiparous cows. J Dairy Sci. 2013;96:4015-25.

41 Kalsner L, Chamberlain SJ. Prader-Willi, Angelman, and 15q11-q13 duplication syndromes. Pediatr Clin N Am. 2015;62:587-606.

42 Driscoll DJ, Miller JL, Schwartz S, et al. Prader-Willi Syndrome. In: Adam MP, Ardinger $\mathrm{HH}$, Pagon RA, et al, editors. GeneReviews ${ }^{\oplus}$. Seattle: University of Washington, Seattle; 1998. pp. 1993-2017. Available from: https://www.ncbi. nlm.nih.gov/books/NBK1330/.

43 Meguro M, Kashiwagi A, Mitsuya K, Nakao M, Kondo I, Saitoh S, et al. A nove maternally expressed gene, ATP10C, encodes a putative aminophospholipid translocase associated with Angelman syndrome. Nat Genet. 2001;28:19-20.

44 DuBose AJ, Johnstone KA, Smith EY, Hallett RAE, Resnick JL. Atp10a, a gene adjacent to the PWS/AS gene cluster, is not imprinted in mouse and is insensitive to the PWS-IC. Neurogenetics. 2010;11:145-51.

45 Schaaf CP, Gonzalez-Garay ML, Xia F, Potocki L, Gripp KW, Zhang B, et al. Truncating mutations of MAGEL2 cause Prader-Willi phenotypes and autism. Nat Genet. 2013;45:1405-8

46 Kaneda M, Takahashi M, Yamanaka K, Saito K, Taniguchi M, Akagi S, et al. Epigenetic analysis of bovine parthenogenetic embryonic fibroblasts. J Repduction Dev. 2017:63:365-75.

47 Schaller F, Watrin F, Sturny R, Massacrier A, Szepetowski P, Muscatelli F. A single postnatal injection of oxytocin rescues the lethal feeding behaviour in mouse newborns deficient for the imprinted Magel2 gene. Hum Mol Genet. 2010;19:4895-905.

48 Schwarzenbacher H, Burgstaller J, Seefried FR, Wurmser C, Hilbe M, Jung S, et al. A missense mutation in TUBD1 is associated with high juvenile mortality in Braunvieh and Fleckvieh cattle. BMC Genomics. 2016;17:400.

49 Armstrong DL, McGowen MR, Weckle A, Pantham P, Caravas J, Agnew D, et al. The core transcriptome of mammalian placentas and the divergence of expression with placental shape. bioRxiv. 2017. http://dx.doi.org/10.1101/ 137554.

50 Pasch E, Link J, Beck C, Scheuerle S, Alsheimer M. The LINC complex component Sun 4 plays a crucial role in sperm head formation and fertility. Biol Open. 2015;4:1792-802.

51 Brew O, Sullivan MHF, Woodman A, Dulay A, Nayeri U, Buhimschi C. Comparison of normal and pre-Eclamptic placental gene expression: a systematic review with meta-analysis. PLoS One. 2016;11:e0161504.

52 Chandra A, van Maldegem F, Andrews S, Neuberger MS, Rada C. Deficiency in spliceosome-associated factor CTNNBL1 does not affect ongoing cell cycling but delays exit from quiescence and results in embryonic lethality in mice. Cell Cycle. 2013;12:732-42.

53 Kappil MA, Green BB, Armstrong DA, Sharp AJ, Lambertini L, Marsit CJ, et al. Placental expression profile of imprinted genes impacts birth weight. Epigenetics. 2015;10:842-9.

\section{Submit your next manuscript to BioMed Central and we will help you at every step:}

- We accept pre-submission inquiries

- Our selector tool helps you to find the most relevant journal

- We provide round the clock customer support

- Convenient online submission

- Thorough peer review

- Inclusion in PubMed and all major indexing services

- Maximum visibility for your research

Submit your manuscript at www.biomedcentral.com/submit

) Biomed Central 